Check for updates

Cite this: RSC Adv., 2017, 7, 19237

Received 8th February 2017

Accepted 25th March 2017

DOI: $10.1039 / \mathrm{c} 7 \mathrm{ra01598d}$

rsc.li/rsc-advances

\section{Controllable synthesis of mesoporous titanosilicates for styrene oxidization using a nanocellulose template strategy $\dagger$}

\begin{abstract}
Dazhi Shen, Jian Liu, (DD * Lihui Gan, Naizhong Huang and Minnan Long*
In this work, mesoporous titanosilicates with tunable pore size were successfully synthesized using green and cheap bio-templates (nanocellulose). A series of samples was systematically prepared, characterized and used as catalysts for styrene oxidation by $\mathrm{H}_{2} \mathrm{O}_{2}$. The results demonstrated that the nanocellulose template strategy reported here was a feasible and effective method. The as-prepared titanosilicates presented high specific surface areas $\left(450-500 \mathrm{~m}^{2} \mathrm{~g}^{-1}\right)$ and their mesopore sizes could be tuned from 2 to $8 \mathrm{~nm}$ by varying the addition amount of templates in the synthesis process. Active tetrahedral $\mathrm{Ti}$ species were efficiently incorporated in the mesoporous silica. Meanwhile, strong acid sites of the samples were confirmed using $\mathrm{NH}_{3}$-TPD. The catalytic results revealed that the optimal catalysts $(\mathrm{Si} / \mathrm{Ti}=$ 20 mol ratio) showed high catalytic activity and product selectivity can be adjusted through changing the mesopore size of the catalysts. The recycling tests indicated that as-prepared catalysts exhibited low catalytic deactivation. In addition, the oxidation mechanism over this catalyst was proposed. Thus, the present novel, sustainable and controllable method showed potential practical applications.
\end{abstract}

\section{Introduction}

Since the discovery of titanosilicate-1 (Ts-1) in 1983, Ti incorporated zeolitic materials have received much attention due to their remarkable catalytic activity, and created an environmentally friendly route for the oxidation of organic compounds using hydrogen peroxide in mild conditions..$^{1,2}$ However, owing to the fact that the pore sizes of conventional microporous titanosilicate zeolites (Ts-1, Ts-2 or other microporous titanosilicates) are smaller than $1 \mathrm{~nm},{ }^{3}$ large organic molecules cannot assess the active sites located inside the cavities which limits the utilization of microporous titanosilicates. To overcome this drawback, mesoporous titanosilicates have been developed and proved to be potential catalysts in a wide range of oxidation reactions, especially for bulky organic molecules oxidation. In recently years, many researches have been conducted on synthesis of Ti containing mesoporous silica, such as, Ti-MCM-type, ${ }^{4-7}$ Ti-HMS-type, ${ }^{8-11}$ Ti-SBA-type, ${ }^{12-16}$ etc., and these catalysts have shown excellent catalytic properties in oxidization reaction including hydroxylation of aromatics, ${ }^{3}$ oxidative desulfurization, ${ }^{17}$ oxidation of alkenes ${ }^{18}$ and so on. Nevertheless, these catalysts suffer from cumbersome synthesis procedures and high cost of templates. Especially for latter, the template agents, such as cetyltrimethyl ammonium bromide,

College of Energy, Xiamen University, Xiamen, 36105, China. E-mail: jianliu@xmu. edu.cn; longmn@xmu.edu.cn; Fax: +86-592-2188053; Tel: +86-592-5952787

$\dagger$ Electronic supplementary information (ESI) available. See DOI: $10.1039 / \mathrm{c} 7 \mathrm{ra0} 1598 \mathrm{~d}$ pluronic triblock copolymer and 1-aminododecanelaurylamine, are all from nonrenewable petroleum-based feed stocks and high cost which limited their large-scale application. Therefore, it is still of great significance to develop a simple, green and cost-effective method by using renewable template materials as an environmentally benign alternative. In this respect, biomaterials, in particular cellulose, attracts special attention as a renewable, inexpensive "green" template. ${ }^{19-21}$

Cellulose is one of the most abundant nature material on the earth, which is sustainable, biodegradable and inexpensive sources. ${ }^{22,23}$ When cellulose fibers are subjected to acid hydrolysis, stable suspensions of nanocellulose are obtained. Nanocellulose is rod-like or ribbon-like object with a length typically ranging from 50 to $1000 \mathrm{~nm}$ and a width varying from 3 to $50 \mathrm{~nm} \cdot{ }^{24-26}$ Due to possessing nanoscale dimension, high specific strength, high surface area, unique nanosize and accessible industrially in large scale, nanocellulose has been considered as a most promising renewable nanomaterials. ${ }^{27-29}$ Recently, nanocellulose has been attracted people's interests in synthesizing mesoporous materials. ${ }^{30-32}$ As a kind of nature biological materials, nanocellulose is different from traditional template agents. In addition, it has high aspect ratio, low density and active surface of hydroxyl groups. Thus it possesses the advantages of traditional soft template and hard template at the same time. ${ }^{33}$

In present work, we demonstrate a facile and sustainable method for the synthesis of titanosilicates with tunable mesopore sizes and high specific surface areas by using nanocellulose as the template for the first time. The characterization 
results revealed that active framework Ti species have effectively incorporated in the mesoporous silicate. Further research illustrated that the pore size of as-synthesized mesoporous titanosilicates was controllable. Finally, oxidation of styrene was adopted as a probe reaction to evaluate the catalysts properties of obtained titanosilicates.

\section{Experimental}

\subsection{Chemical and materials}

Cellulose from absorbent cotton, sulphuric acid, tetraethylorthosilicate (TEOS), tetrabutyl orthotitanate (ТBOT), acetylacetone, styrene, hydrogen peroxide $(30 \% \mathrm{w} / \mathrm{v})$, acetonitrile. All reagents was purchased from Shanghai Chemical Reagent Co. Ltd. (Shanghai, China) and used as received without further purification.

\subsection{Synthesis of titanosilicates}

Suspensions of nanocellulose (NCC) were prepared as the previous literature ${ }^{34}$ with slight modification. In typical, ten gram of cotton was treated with $100 \mathrm{ml}$ of $65 \%(\mathrm{w} / \mathrm{w}) \mathrm{H}_{2} \mathrm{SO}_{4}$ at $45{ }^{\circ} \mathrm{C}$ for 120 min under vigorous stirring. After that, the obtained nanocellulose suspension was diluted for 5 -fold with deionized water to terminate the hydrolysis. The final aqueous suspensions were sonicated for $20 \mathrm{~min}$. The final aqueous suspensions were approximately $1.2 \%$ NCC concentration by weight.

One-pot synthesized titanium-silicate with various Ti loading and pore size. In typically, $20 \mathrm{ml}$ of ethanol, $10 \mathrm{ml}$ TEOS, specified mixture of acetylacetone and TBOT (molar ratio of acetylacetone/ТВOT $=1 / 1$ ) were thoroughly mixed at room temperature. The molar composition of the mixture was 1 TEOS: $0.01-0.1$ ТВOT: $0.01-0.1$ acetylacetone: 7.2 ethanol. To this solution, specified volume of as-prepared nanocellulose suspensions $(1.2 \% \mathrm{wt})$ were added with vigorous stirring. The final solution was further stirred at room temperature for $2 \mathrm{~h}$, and the resulting mixture formed a gel in an oven at $100{ }^{\circ} \mathrm{C}$ for $10 \mathrm{~h}$. Finally, the product obtained was washed with deionized water and calcined at $550{ }^{\circ} \mathrm{C}$ for $5 \mathrm{~h}$ in air to remove the biotemplate incorporated in the composites. After that, the obtained samples were crushed in an agate mortar. As a result, the samples obtained were denoted as Ts- $X(Y)$, where $X=10,20,60$ or 100 corresponding to the Si/Ti molar ratio and $Y=0.5,1.5$ or 3.0 corresponding to the volume ratio of nanocellulose suspensions to TEOS.

\subsection{Characterization}

Transmission electron microscopy (TEM) images were obtained on Zeiss Libra200FE operated at $200 \mathrm{kV}$. XRD spectra of catalysts were acquired on a Rigaku Ultima IV X-ray diffractometer with Ni-filtered $\mathrm{Cu} \mathrm{K} \alpha$ radiation operated at $40 \mathrm{kV}$ and $30 \mathrm{~mA}$. The data was collected from $2 \theta=10^{\circ}$ to $90^{\circ}$ at a scan rate of $10^{\circ}$ $\mathrm{min}^{-1}$. The nitrogen adsorption and desorption isotherms were measured on an ASAP 2020 system in static measurement mode. The samples were degassed at $300{ }^{\circ} \mathrm{C}$ for $4 \mathrm{~h}$ before the measurement. The specific surface area of sample was calculated by BET model. The pore size distribution curves were obtained by the BJH (Barrett-Joyner-Halenda) method based on the adsorption branch of isotherm. The diffuse reflectance UVvis spectra were recorded on a VARIAN Cary 5000 spectrometer equipped with a diffuse reflectance attachment and using $\mathrm{BaSO}_{4}$ as a reference. FT-IR spectra were recorded on a Nicolet is5 spectrometer. $\mathrm{NH}_{3}$-TPD was measured by using a Micromeritics AutoChem II 2920 equipped with a thermal conductivity detector (TCD).

\subsection{Catalytic activity}

The catalytic tests were performed in a sealed round bottom flask in which vigorous stirring at $80^{\circ} \mathrm{C}$. Typically, the reactor was charged with $200 \mu \mathrm{l}$ styrene, $2 \mathrm{ml}$ of acetonitrile as solvent, specified hydrogen peroxide and $0.08 \mathrm{~g}$ catalyst loading. The oxidation products were analyzed by gas chromatography on Agilent 7890. After the reactions, the catalyst was separated by centrifugation, washed with acetonitrile for three times and dried at $100{ }^{\circ} \mathrm{C}$ for $6 \mathrm{~h}$ to be reused.

\section{Results and discussion}

\subsection{Catalyst characteristics}

Fig. 1 shows the XRD spectra of the samples with different $\mathrm{Si} / \mathrm{Ti}$ molar ratio. As seen, all the curves show a relatively weak diffraction band at the $2 \theta$ range of $15-35^{\circ}$, which is associated with the amorphous silica materials. ${ }^{15,35}$ Additional peaks characteristic of $\mathrm{TiO}_{2}$ are not detected in all samples. The result indicates that the separate $\mathrm{TiO}_{2}$ phase is absent in the samples.

UV-vis diffuse reflectance spectroscopy has been used to study the environment of titanium in amorphous silica in the wavelength range of $200 \mathrm{~nm}$ to $800 \mathrm{~nm}$. As seen in Fig. 2, the maxima of absorption band of all samples center at $220 \mathrm{~nm}$ together with a weak shoulder band at $270 \mathrm{~nm}$. The band at $220 \mathrm{~nm}$ can be attributed to tetrahedral Ti species and the shoulder band at $270 \mathrm{~nm}$ indicate the presence of titanium in an octahedral coordination environment. ${ }^{6}$ Absorbance value of two bands suggests that a major of titanium species presents in tetrahedral and accompanies by a less fraction in octahedral coordination. ${ }^{3}$ Moreover, the absorption bands are not detected in the range of 350-400 $\mathrm{nm}$ indicating that the presence of a free $\mathrm{TiO}_{2}$ clusters or extra-framework anatase $\mathrm{TiO}_{2}$ species in all

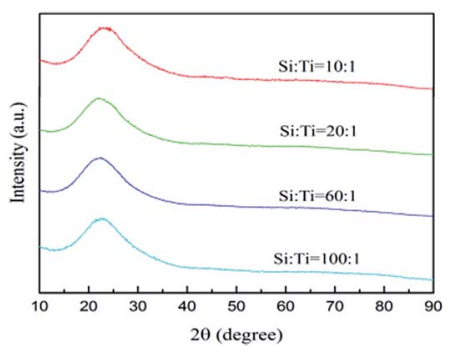

Fig. 1 XRD patterns of titanosilicates synthesized with different Si/Ti molar ratio. 

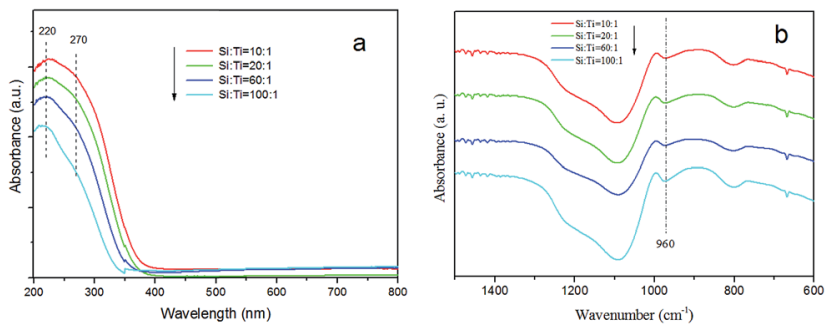

Fig. 2 (a) UV-vis spectra and (b) FT-IR spectra of titanosilicates with different Si/Ti mole ratios.

samples can be ruled out, ${ }^{17}$ which are in good agreement with the XRD results.

The FT-IR spectra of the samples are shown in Fig. 2b. All samples appear a band at $960 \mathrm{~cm}^{-1}$ which can be associated with the framework $\mathrm{Si}-\mathrm{O}-\mathrm{Ti}$ vibrations. ${ }^{2}$ The existence of $\mathrm{Si}-\mathrm{O}-$ $\mathrm{Ti}$ indicates that the $\mathrm{Ti}$ species are incorporation into the framework which would supply active sites on catalyst. The result further confirms that active framework Ti species have efficiently incorporated in the mesoporous silica.

In this work, the samples have been synthesized under acidic precursor solution conditions which is from the acidic nanocellulose suspension solution. The $\mathrm{pH}$ of the synthesis precursor solutions are in the range of $0.2-0.4$. The silica/Ti precursor will hydrolysis under the action of acid catalyst. ${ }^{36}$ However, it is well known that the hydrolysis of silica precursor (TEOS) is slower than that of titanium precursor (TBOT). To avoid the incongruous hydrolysis rates, acetylacetone has been used as a chelating agent to ensure active framework Ti species efficiently incorporating into the framework of silica rather than separate $\mathrm{TiO}_{2}$ crystals.

Fig. 3a and $\mathrm{b}$ show the $\mathrm{N}_{2}$ adsorption-desorption isotherms and the pore size distribution for the samples with different $\mathrm{Si} /$
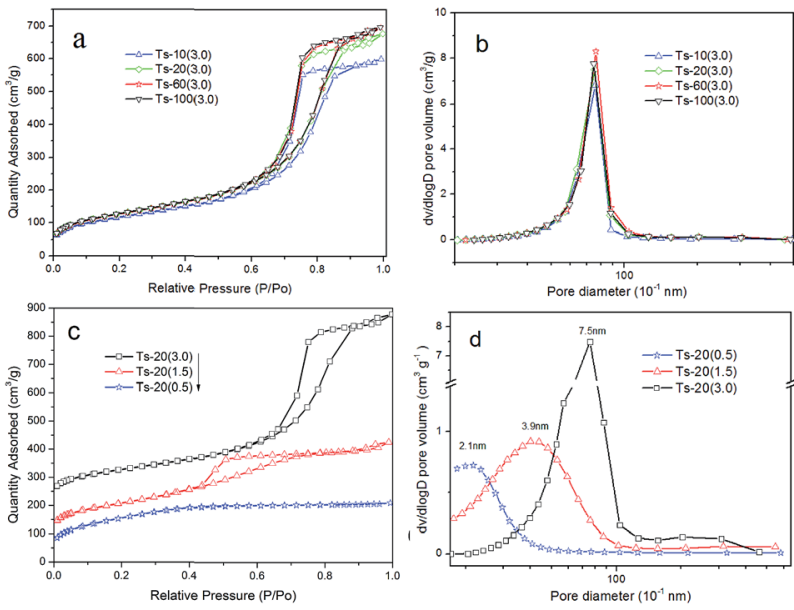

Fig. $3 \mathrm{~N}_{2}$ adsorption and desorption isotherms of mesoporous titanosilicates with different $\mathrm{Si} / \mathrm{Ti}$ ratio (a), corresponding pore size distribution (b), different CNC/TEOS ratio (c) and corresponding pore size distribution (d). The isotherms for products Ts-20(1.5) and Ts20 (30) were vertically offset by 70 and $200 \mathrm{~cm}^{3} \mathrm{~g}^{-1}$, respectively in part (c).
Ti ratios. As can be seen, all products show type-IV curves ${ }^{37}$ with a narrow distribution of mesoporous diameters centering at about $7.5 \mathrm{~nm}$. Specific surface areas and pore volume change slight at $\mathrm{Si} / \mathrm{Ti}=100,60$ and 20 while they decrease prominently at $\mathrm{Si} / \mathrm{Ti}=10$. These changes could be observed clearly in Table 1 (entry 1-4). This result indicates that titanium incorporated in $\mathrm{SiO}_{2}$ framework has little effect on the mesoporous structure at high Si/Ti ratio. However, excessive-loading of titanium $(\mathrm{Si} / \mathrm{Ti}=$ 10 ) in the sample could also induce a reduction in BET surface area and pore volume.

The influence of templates ratio on the morphology and pore structure has been investigated. The results are showed in Fig. $3 \mathrm{c}$ and d, and the corresponding parameters are listed in Table 1 (entry $5-7$ ). The pore diameter and pore volume significantly increase with the addition amount of nanocellulose templates in precursor solution. Subsequently, the specific surface areas of the samples slightly decrease. These results confirm that the pore size of the catalysts can be systematically adjusted by varying the amounts of bio-template (nanocellulose) in the synthesis system.

Fig. 4 gives the TEM images of as-prepared samples with the NCC/TEOS ratio at $0.5,1.5$ and 3, respectively. The synthesized samples show the patterns characteristic of disorder wormholelike meso-structure. The similar structure was also observed by previously reports. ${ }^{38,39}$

The property of acid sites is determined by $\mathrm{NH}_{3}$-TPD (Fig. 5). Two major desorption peaks are observed at $150-250{ }^{\circ} \mathrm{C}$ and $300-800{ }^{\circ} \mathrm{C}$ in Fig. 5 . The former can be attributed to ammonia desorbed from weaker acid centers, and the latter was from relatively stronger acid centers. The acidic sites of the samples can be attributed the unsaturated $\mathrm{Ti}^{4+}$ species at surface defect sites of the catalysts. ${ }^{15,40}$

\subsection{Catalytic performances of catalysts}

3.2.1 Effect of $\mathrm{Si}$ /Ti ratio. Finally, the catalytic performance of as-synthesized samples are evaluated through oxidation of styrene by using $30 \mathrm{wt} \% \mathrm{H}_{2} \mathrm{O}_{2}$ as an oxidant. The main products of the reaction are benzaldehyde, 1-phenyl-1,2-ethanediol and benzoic acid. The effect of catalysts in different $\mathrm{Si} / \mathrm{Ti}$ mole ratio catalysts is investigated and the results are presented in Fig. 6a. Obviously, the catalytic activity (styrene conversion) increases firstly with the increasing of Ti content but then drop. The optimum result is at $\mathrm{Si} / \mathrm{Ti}=20$ with the highest conversion of $71.3 \%$. It is reasonable that the activity site increase with the $\mathrm{Ti}$ content. However, the catalytic activity does not increase at $\mathrm{Si} / \mathrm{Ti}$ $=10$. The result may be mainly due to the decreased specific surface areas of the sample (Table 1, entry 1 ), which reduce the activity sites on the surface of the catalysts.

3.2.2 Effect of pore size. Catalysts with three different pore sizes at the $\mathrm{Si} / \mathrm{Ti}=20$ were evaluated and the results are shown in Fig. 6b. The catalysts Ts-20(0.5) and Ts-20(1.5) exhibit almost the same conversions $(73.5 \%$ and $74.4 \%$, respectively) which can be attributed to their similar specific surface areas. Owing to the relative low specific surface areas of Ts-20(3.0) compared with the Ts-20(0.5) and Ts-20(1.5), the conversion correspondingly decrease to $71.3 \%$. As for the effects of pore size on 
Table 1 Textural characterization of as-prepared titanosilicates

\begin{tabular}{|c|c|c|c|c|c|c|}
\hline Entry & Cat. & $\begin{array}{l}\mathrm{Si} / \mathrm{Ti} \\
\text { (mole ratio) }\end{array}$ & $\begin{array}{l}\text { NCC/TEOS } \\
(\mathrm{v} / \mathrm{v})\end{array}$ & $\begin{array}{l}S \\
\left(\mathrm{~m}^{2} \mathrm{~g}^{-1}\right)\end{array}$ & $\begin{array}{l}\mathrm{DBJH}^{a} \\
(\mathrm{~nm})\end{array}$ & $\begin{array}{l}V_{\mathrm{p}}^{b} \\
\left(\mathrm{~cm}^{3} \mathrm{~g}^{-1}\right)\end{array}$ \\
\hline 1 & Ts-10(3.0) & 10 & 3 & 425.1 & 7.6 & 0.93 \\
\hline 3 & Ts-60(3.0) & 60 & 3 & 462.6 & 7.5 & 1.09 \\
\hline 4 & Ts-100(3.0) & 100 & 3 & 466.2 & 7.4 & 1.08 \\
\hline 5 & Ts-20(0.5) & 20 & 0.5 & 516.9 & 2.1 & 0.32 \\
\hline
\end{tabular}

${ }^{a}$ Pore diameter (obtained from the adsorption isotherm by the $\mathrm{BJH}$ method). ${ }^{b}$ Pore volume determined by nitrogen adsorption at a relative pressure of 0.98 .
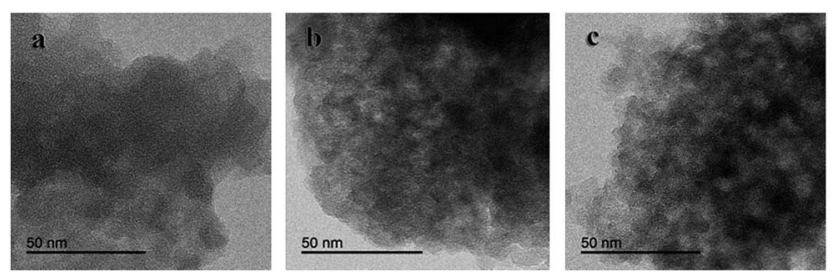

Fig. 4 TEM images of the samples. Ts-20(0.5) (a), Ts-20(1.5) (b) and Ts-20(3.0) (c).

products distribution, the Ts-20(3.0) with the pore size at $7.5 \mathrm{~nm}$ gave a better performance in selectivity for benzaldehyde than other catalysts. Whereas, the Ts-20(0.5) with the pore size at $2.1 \mathrm{~nm}$ show a better selectivity for 1-phenyl-1,2-ethanediol. This result indicates that different pore size of the catalysts has an obvious effect on the reaction. Thus, as-prepared catalysts with tunable pore size will be advantageous to adjust the selectivity of the products.

3.2.3 Effect of $\mathbf{H}_{2} \mathrm{O}_{2} /$ styrene. The sample Ts-20(3.0) was chosen to investigate the influences of $\mathrm{H}_{2} \mathrm{O}_{2}$ /styrene mole ratio and the results are shown in Fig. 6c. It is obvious that an increased oxidant/reactant ratio leads to the facilitation of styrene conversion. With the $\mathrm{H}_{2} \mathrm{O}_{2} /$ styrene ratio increasing from 1.5 to 3 , the conversion of styrene increase from $63.5 \%$ to $86.3 \%$. But simultaneously, the selectivity for 1-phenyl-1,2ethanediol decrease obviously from $24.9 \%$ to $15.3 \%$ and, benzaldehyde and benzoic acid selectivity rise.

3.2.4 Effect of reaction time. Fig. $6 \mathrm{~d}$ showed the effect of reaction time on styrene oxidation using Ts-20(3.0). It was found that styrene conversion increased rapidly from 46.3 to

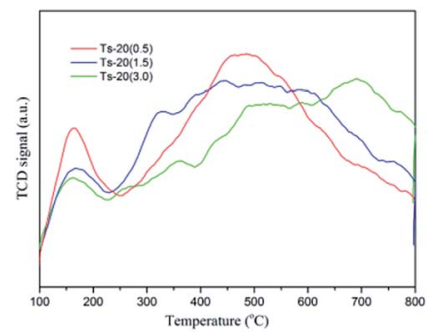

Fig. $5 \mathrm{NH}_{3}$-TPD spectra of Ts-20(0.5); Ts-20(1.5); Ts-20(3.0).
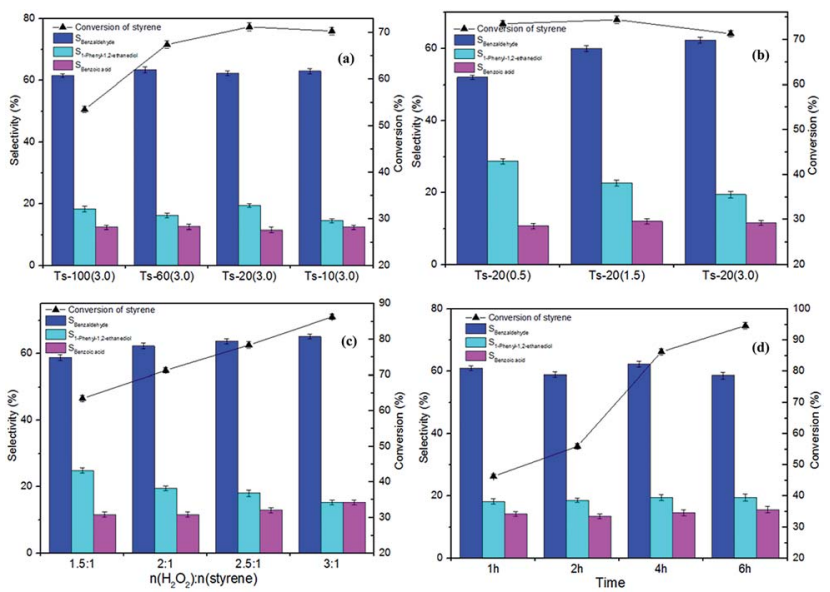

Fig. 6 Catalytic performances on the oxidization of styrene. (a) Effect of $\mathrm{Ti} / \mathrm{Si}$ ratio $\left(80^{\circ} \mathrm{C}, 4 \mathrm{~h}, \mathrm{H}_{2} \mathrm{O}_{2} /\right.$ styrene $\left.=2\right)$; (b) effect of pore size of catalysts $\left(80^{\circ} \mathrm{C}, 4 \mathrm{~h}, \mathrm{H}_{2} \mathrm{O}_{2} /\right.$ styrene $\left.=2\right)$; (c) effect of the $\mathrm{H}_{2} \mathrm{O}_{2}$ /styrene (catalyst: Ts-20(3.0), $80{ }^{\circ} \mathrm{C}, 4 \mathrm{~h}$ ); (d) effect of reaction time (catalyst: Ts-20(3.0), $80{ }^{\circ} \mathrm{C}, \mathrm{H}_{2} \mathrm{O}_{2}$ /styrene $=3$ ).

$86.3 \%$ with prolongation of reaction time from 1 to $4 \mathrm{~h}$. Only little increment was observed when reaction time was prolonged to $6 \mathrm{~h}$. Moreover, the product distribution showed little changes with reaction time.

3.2.5 Proposed mechanism of oxidation reaction. It is well accepted that the oxidation of styrene at the side chain can obtain different products depending on the catalysts and reaction conditions. According to previous reports, ${ }^{41-43}$ we proposed that the reaction process may have two major reaction routes (Scheme 1). On the presence of catalysts, benzaldehyde, 1phenyl-1,2-ethanediol and benzoic acid are detected in the oxidation products. The main product is benzaldehyde, which is from the direct oxidative cleavage of the styrene side chain double bond ${ }^{40}$ (route 1 , Scheme 1). Benzaldehyde can also be from a oxidization of epoxystyrene (route $2-1$, Scheme 1 ), and it is also readily oxidized to benzoic acid. ${ }^{41}$ It is interesting that epoxystyrene is not detected in products compared with previous reports. ${ }^{2,18}$ This can be ascribed to the existence of acid site on our catalysts and the subsequent hydrolysis of epoxy group. $^{44}$ Therefore, the product of 1-phenyl-1,2-ethanediol is derived from the hydrolysis of the epoxystyrene (route 2-2, Scheme 1). Furthermore, there is a competition between the 


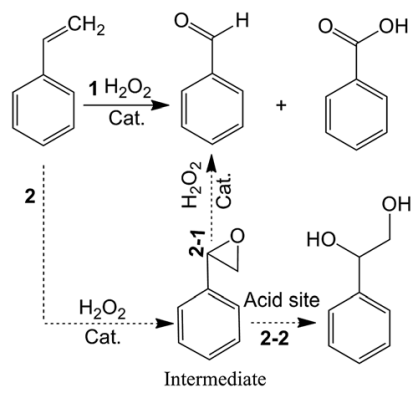

Scheme 1 Proposed reaction mechanism of styrene oxidation

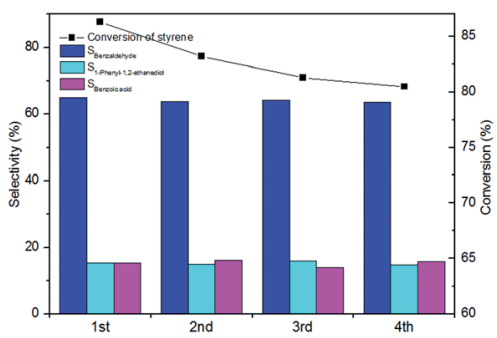

Fig. 7 Catalyst reusability (Ts-20(3.0); $\mathrm{H}_{2} \mathrm{O}_{2}$ /styrene $=3 ; 80{ }^{\circ} \mathrm{C} ; 4$ h.)

oxidation (route 2-1) and hydrolysis of epoxystyrene (route 2-2, Scheme 1), which is in good agreement with our experimental results that if more oxidant $\left(\mathrm{H}_{2} \mathrm{O}_{2}\right)$ is employed in the reaction, the selectivity of benzaldehyde increases, while the selectivity of 1-phenyl-1,2-ethanediol decreases.

It is noted that pore sizes of the catalysts have a prominent effect on product selectivity in this work. This phenomenon can be attributed to the effect of diffusion of the reaction species in different catalysts pore size. The catalysts (Ts-20(3.0)) with macropores tend to oxidization reaction for the fast diffusion of reactants in the pores. Thus, the catalysts (Ts-20(3.0)) have higher selectivity for oxidation product than the others. In contrast, narrow pore channel on the catalysts (Ts-20(0.5)) inhibits further oxidization of the intermediate (epoxystyrene) owing to the poor diffusion which will cause more hydrolysis of intermediate (epoxystyrene, route $2-2$, Scheme 1 ) on the acid site of the catalysts. Thus, the catalyst (Ts-20(0.5)) has the best selectivity for hydrolysis product (1-phenyl-1,2-ethanediol).

3.2.6 Catalyst reusability. In order to characterize the durability of the catalysts, the recycling of the catalyst was carried out. The catalysts (Ts-20(3.0)) was chosen and evaluated in four recycling experiments. As seen from Fig. 7, the catalyst is quite stable even in the 4 th recycle. The conversion is still remained $80 \%$ and little variation in products selectivity is detected. Furthermore, we find that this catalyst (Ts-20(3.0)) gave competitive activity compared with previous reports (see the ESI, Table S1†).

\section{Conclusions}

In this article, we demonstrated a novel, facile and controllable method for synthesis of mesoporous titanosilicates by using bio-template (nanocellulose) for the first time. As-prepared titanosilicates are proved with high surface areas $\left(450-500 \mathrm{~m}^{2}\right.$ $\left.\mathrm{g}^{-1}\right)$ and tunable pore size $(2-8 \mathrm{~nm})$. Active framework Ti has effectively incorporated in the frameworks of silicate. Meanwhile, strong acid sites of the samples have been confirmed by $\mathrm{NH}_{3}$-TPD. The catalyst tests illustrate that the optimum $\mathrm{Si} / \mathrm{Ti}$ atom ratio is 20 and product selectivity can be adjusted through changing the mesopore size of the catalysts. The recycling tests indicate that the catalysts exhibit low catalytic deactivation. Hence, the catalysts synthesized with presenting method will be advantageous to general applications in various molecular catalysis due to their tunable pore size.

\section{Acknowledgements}

This work was supported by the National Natural Science Foundation of China (No. 21303142), the research fund from the Xiamen Southern Oceanographic Center (No. 4GZP59HJ29), Fujian Provincial Department of Ocean and Fisheries (No. 201425), and President Fund of Xiamen University (No. 20720150090).

\section{References}

1 F. Jin, S. Y. Chen, L. Y. Jang, J. F. Lee and S. F. Cheng, J. Catal., 2014, 319, 247-257.

2 J. J. Wang, J. M. Lu, J. H. Yang, R. Chen, Y. Zhang, D. H. Yin and J. Q. Wang, Appl. Surf. Sci., 2013, 283, 794-801.

3 A. Kumar and D. Srinivas, J. Mol. Catal. A: Chem., 2013, 368, 112-118.

4 R. Peng, D. Zhao, N. M. Dimitrijevic, T. Rajh and R. T. Koodali, J. Phys. Chem. C, 2012, 116, 1605-1613.

5 J. Silvestre-Albero, M. E. Domine, J. L. Jorda, M. T. Navarro, F. Rey, F. Rodriguez-Reinoso and A. Corma, Appl. Catal., A, 2015, 507, 14-25.

6 J. M. Fraile, N. García, J. A. Mayoral, F. G. Santomauro and M. Guidotti, ACS Catal., 2015, 5, 3552-3561.

7 K. F. Lin, P. P. Pescarmona, H. Vandepitte, D. Liang, G. Van Tendeloo and P. A. Jacobs, J. Catal., 2008, 254, 64-70.

8 Y. Wang, G. Li, X. S. Wang and C. Z. Jin, Energy Fuels, 2007, 21, 1415-1419.

9 B. Pawelec, J. L. G. Fierro, A. Montesinos and T. A. Zepeda, Appl. Catal., B, 2008, 80, 1-14.

10 T. A. Zepeda, A. Infantes-Molina, J. N. D. de Leon, R. ObesoEstrella, S. Fuentes, G. Alonso-Nunez and B. Pawelec, J. Mol. Catal. A: Chem., 2015, 397, 26-35.

11 Y. B. Zhuang, H. Y. Song, G. Li and Y. J. Xu, Mater. Lett., 2010, 64, 2491-2493.

12 S. D. Shen, Y. Deng, G. B. Zhu, D. S. Mao, Y. H. Wang, G. S. Wu, J. Li, X. Z. Liu, G. Z. Lu and D. Y. Zhao, J. Mater. Sci., 2007, 42, 7057-7061.

13 F. Berube, B. Nohair, F. Kleitz and S. Kaliaguine, Chem. Mater., 2010, 22, 1988-2000.

14 R. V. Sharma, K. K. Soni and A. K. Dalai, Catal. Commun., 2012, 29, 87-91.

15 A. Kumar and D. Srinivas, J. Catal., 2012, 293, 126-140.

16 S.-Y. Chen, T. Mochizuki, Y. Abe, M. Toba and Y. Yoshimura, Applied Catalysis B: Environmental, 2014, 148-149, 344-356. 
17 T. W. Kim, M. J. Kim, F. Kleitz, M. M. Nair, R. Guillet-Nicolas, K. E. Jeong, H. J. Chae, C. U. Kim and S. Y. Jeong, Chemcatchem, 2012, 4, 687-697.

18 M. Fadhli, I. Khedher and J. M. Fraile, J. Mol. Catal. A: Chem., 2016, 420, 282-289.

19 S. J. Eichhorn, A. Dufresne, M. Aranguren, N. E. Marcovich, J. R. Capadona, S. J. Rowan, C. Weder, W. Thielemans, M. Roman, S. Renneckar, W. Gindl, S. Veigel, J. Keckes, H. Yano, K. Abe, M. Nogi, A. N. Nakagaito, A. Mangalam, J. Simonsen, A. S. Benight, A. Bismarck, L. A. Berglund and T. Peijs, J. Mater. Sci., 2010, 45, 1-33.

20 P. Tingaut, T. Zimmermann and G. Sebe, J. Mater. Chem., 2012, 22, 20105-20111.

21 D. Klemm, F. Kramer, S. Moritz, T. Lindstrom, M. Ankerfors, D. Gray and A. Dorris, Angew. Chem., 2011, 50, 5438-5466.

22 Y. Hu, L. Tang, Q. Lu, S. Wang, X. Chen and B. Huang, Cellulose, 2014, 21, 1611-1618.

23 H.-M. Ng, L. T. Sin, T.-T. Tee, S.-T. Bee, D. Hui, C.-Y. Low and A. R. Rahmat, unpublished work.

24 M. Kaushik and A. Moores, Green Chem., 2016, 18, 622-637. 25 Q. Q. Li, S. McGinnis, C. Sydnor, A. Wong and S. Renneckar, ACS Sustainable Chem. Eng., 2013, 1, 919-928.

26 M. C. Li, Q. L. Wu, K. L. Song, S. Lee, Y. Qing and Y. Q. Wu, ACS Sustainable Chem. Eng., 2015, 3, 821-832.

27 K. Oksman, Y. Aitomäki, A. P. Mathew, G. Siqueira, Q. Zhou, S. Butylina, S. Tanpichai, X. J. Zhou and S. Hooshmand, Composites, Part A, 2016, 83, 2-18.

28 N. Lavoine, I. Desloges, A. Dufresne and J. Bras, Carbohydr. Polym., 2012, 90, 735-764.

29 H. Y. Yu, Z. Y. Qin, C. F. Yan and J. M. Yao, ACS Sustainable Chem. Eng., 2014, 2, 875-886.
30 B. Paredes, A. Widera, V. Murg, O. Mandel, S. Folling, I. Cirac, G. V. Shlyapnikov, T. W. Hansch and I. Bloch, Nature, 2004, 429, 277-281.

31 E. Dujardin, M. Blaseby and S. Mann, J. Mater. Chem., 2003, 13, 696-699.

32 K. E. Shopsowitz, H. Qi, W. Y. Hamad and M. J. Maclachlan, Nature, 2010, 468, 422-425.

33 F. Shi, T. Yu, S.-C. Hu, J.-X. Liu, L. Yu and S.-H. Liu, Chem. Eng. J., 2016, 292, 105-112.

34 S. Beck-Candanedo, M. Roman and D. G. Gray, Biomacromolecules, 2005, 6, 1048-1054.

35 S. Y. Chen, T. Mochizuki, Y. Abe, M. Toba and Y. Yoshimura, Appl. Catal., B, 2014, 148, 344-356.

36 M. A. Betiha, H. M. A. Hassan, A. M. Al-Sabagh, A. S. Khder and E. A. Ahmed, J. Mater. Chem., 2012, 22, 17551-17559.

37 H. L. Chen, Y. P. Peng, K. F. Chen, C. H. Lai and Y. C. Lin, J. Environ. Sci., 2016, 44, 76-87.

38 T. Witoon, M. Chareonpanich and J. Limtrakul, Mater. Lett., 2008, 62, 1476-1479.

39 M. A. Betiha, S. A. Mahmoud, M. F. Menoufy and A. M. AlSabagh, Appl. Catal., B, 2011, 107, 316-326.

40 S.-Y. Chen, T. Mochizuki, Y. Abe, M. Toba and Y. Yoshimura, Appl. Catal., B, 2014, 148-149, 344-356.

41 V. Hulea and E. Dumitriu, Appl. Catal., A, 2004, 277, 99-106. 42 A. D. S. Neto, L. G. Pinheiro, J. M. Filho and A. C. Oliveira, Fuel, 2015, 150, 305-317.

43 X. C. Zhu, R. W. Shen and L. X. Zhang, Chin. J. Catal., 2014, 35, 1716-1726.

44 N. T. Thao and L. T. K. Huyen, Chem. Eng. J., 2015, 279, 840850. 